\title{
Erratum to: Increased In-hospital Complications After Primary Posterior versus Primary Anterior Cervical Fusion
}

\author{
Stavros G. Memtsoudis MD, PhD, Alexander Hughes MD, \\ Yan Ma PhD, Ya Lin Chiu MS, Andrew A. Sama MD, \\ Federico P. Girardi MD
}

Published online: 20 February 2011

(C) The Association of Bone and Joint Surgeons ß 2011

\section{Erratum to: Clin Orthop Relat Res DOI 10.1007/s11999-010-1549-4}

The aforementioned article contains incorrect information in three of the cells in Tables 1 and 2. The errors occurred as a consequence of an oversight in reporting frequencies with a count of equal or less than 10 in any individual table cell, which is against rules put forth by the Healthcare Cost and Utilization Project. Cells containing such errors were those presenting unweighted frequencies of "Discharge Status: Alive, destination unknown" for anterior and posterior surgeries in Table 1, and the unweighted frequency of "Shock" for posterior surgery in Table 2. The corrected tables appear below.

The online version of the original article can be found under doi: 10.1007/s11999-010-1549-4.

S. G. Memtsoudis $(\square)$

Department of Anesthesiology, Hospital for Special Surgery,

Weill Medical College of Cornell University, 535 East

70thStreet, New York, NY 10021, USA

e-mail: MemtsoudisS@hss.edu

Y. Ma, Y. L. Chiu

Department of Public Health and Biostatistics, Hospital for Special Surgery, Weill Medical College of Cornell University, New York, NY, USA

\author{
A. Hughes, A. A. Sama, F. P. Girardi \\ Department of Orthopaedic Surgery, Division of Spine Surgery, \\ Hospital for Special Surgery, Weill Medical College of Cornell \\ University, New York, NY, USA \\ F. P. Girardi \\ e-mail: girardif@hss.edu
}


Table 1. Demographics of anterior and posterior cervical spine fusion surgery discharges

\begin{tabular}{|c|c|c|c|c|c|c|c|}
\hline \multirow[t]{2}{*}{ Patient demographics } & \multicolumn{3}{|l|}{ Anterior } & \multicolumn{3}{|c|}{ Posterior } & \multirow[t]{2}{*}{ p Value } \\
\hline & $\mathrm{F}$ & WF & Percent & $\mathrm{F}$ & WF & Percent & \\
\hline Frequency/weighted frequency/percent & 209,136 & $1,014,778$ & 91.68 & 18,977 & 93,227 & 8.32 & \\
\hline Age groups (years) & & & & & & & $<0.0001$ \\
\hline $0-44$ & 74,244 & 361,346 & 35.71 & 5195 & 25,618 & 28.00 & \\
\hline $45-64$ & 109,137 & 528,541 & 52.24 & 7896 & 38,685 & 42.29 & \\
\hline $65-74$ & 18,588 & 89,982 & 8.89 & 3334 & 16,342 & 17.86 & \\
\hline $75+$ & 6563 & 31,949 & 3.16 & 2199 & 10,833 & 11.84 & \\
\hline Gender & & & & & & & $<0.0001$ \\
\hline Male & 103,051 & 500,973 & 49.40 & 11,161 & 54,820 & 58.73 & \\
\hline Female & 105,969 & 513,231 & 50.60 & 7840 & 38,522 & 41.27 & \\
\hline Race & & & & & & & $<0.0001$ \\
\hline White & 124,969 & 604,899 & 84.03 & 11,224 & 55,154 & 78.06 & \\
\hline Black & 12,617 & 60,789 & 8.44 & 1697 & 8287 & 11.73 & \\
\hline Hispanic & 6369 & 30,305 & 4.21 & 880 & 4251 & 6.02 & \\
\hline Other & 4951 & 23,890 & 3.32 & 602 & 2967 & 4.20 & \\
\hline Insurance & & & & & & & $<0.0001$ \\
\hline Medicare & 34,702 & 168,000 & 16.60 & 6416 & 31,479 & 33.78 & \\
\hline Medicaid & 10,316 & 50,495 & 4.99 & 1501 & 7395 & 7.93 & \\
\hline Private/HMO & 131,200 & 636,735 & 62.92 & 8779 & 43,249 & 46.40 & \\
\hline Other & 32,341 & 156,731 & 15.49 & 2276 & 11,077 & 11.89 & \\
\hline Discharge status & & & & & & & $<0.0001$ \\
\hline Routine & 192,569 & 933,813 & 92.02 & 11,632 & 56,992 & 61.13 & \\
\hline Short-term hospital & 502 & 2444 & 0.24 & 251 & 1248 & 1.34 & \\
\hline Other transfers & 8958 & 44,121 & 4.35 & 5072 & 25,078 & 26.90 & \\
\hline Home health care & 6451 & 31,170 & 3.07 & 1731 & 8467 & 9.08 & \\
\hline Against medical advice & 113 & 549 & 0.05 & 14 & 69 & 0.07 & \\
\hline Died in hospital & 533 & 2632 & 0.26 & 272 & 1347 & 1.45 & \\
\hline Alive, destination unknown & $\leq 10$ & 50 & 0.00 & $\leq 10$ & 25 & 0.03 & \\
\hline Hospital size & & & & & & & $<0.0001$ \\
\hline Small & 20,472 & 93,708 & 9.23 & 1479 & 6860 & 7.34 & \\
\hline Medium & 52,135 & 248,759 & 24.51 & 3777 & 18,295 & 19.58 & \\
\hline Large & 136,529 & 672,333 & 66.25 & 13,766 & 68,289 & 73.08 & \\
\hline Hospital location & & & & & & & $<0.0001$ \\
\hline Rural & 10,242 & 50,632 & 4.99 & 610 & 3047 & 3.26 & \\
\hline Urban & 198,894 & 964,169 & 95.01 & 18,412 & 90,398 & 96.74 & \\
\hline Teaching status & & & & & & & $<0.0001$ \\
\hline Nonteaching & 98,550 & 467,095 & 46.03 & 5623 & 26,834 & 28.72 & \\
\hline Teaching & 110,586 & 547,706 & 53.97 & 13,399 & 66,611 & 71.28 & \\
\hline Admission type & & & & & & & $<0.0001$ \\
\hline Emergency & 11,799 & 58,188 & 6.32 & 3875 & 19,208 & 22.83 & \\
\hline Urgent & 14,770 & 71,013 & 7.71 & 1749 & 8590 & 10.21 & \\
\hline Elective & 162,539 & 789,486 & 85.75 & 11,346 & 55,633 & 66.14 & \\
\hline
\end{tabular}

$\mathrm{F}=$ frequency; $\mathrm{WF}=$ weighted frequency. 
Table 2. Procedure-related complications after spine fusion

\begin{tabular}{|c|c|c|c|c|c|c|c|}
\hline \multirow[t]{2}{*}{ Complications } & \multicolumn{3}{|c|}{ Anterior } & \multicolumn{3}{|c|}{ Posterior } & \multirow[t]{2}{*}{ p Value } \\
\hline & $\mathrm{F}$ & WF & Percent & $\mathrm{F}$ & WF & Percent & \\
\hline Device-related complications & 2410 & 11671 & 1.15 & 2410 & 11671 & 6.92 & $<0.0001$ \\
\hline \multicolumn{8}{|l|}{ Organ-specific complications } \\
\hline Central nervous system & 494 & 2415 & 0.24 & 151 & 754 & 0.81 & $<0.0001$ \\
\hline Cardiac & 629 & 3080 & 0.3 & 193 & 962 & 1.03 & $<0.0001$ \\
\hline Peripheral vascular & 46 & 233 & 0.02 & 19 & 96 & 0.1 & $<0.0001$ \\
\hline Respiratory & 1079 & 5271 & 0.52 & 290 & 1455 & 1.56 & $<0.0001$ \\
\hline Gastrointestinal & 708 & 3455 & 0.34 & 118 & 578 & 0.62 & $<0.0001$ \\
\hline Genitourinary & 855 & 4214 & 0.42 & 214 & 1065 & 1.14 & $<0.0001$ \\
\hline \multicolumn{8}{|l|}{ Other complications of procedure } \\
\hline Shock & 16 & 77 & 0.01 & $\leq 10$ & 34 & 0.04 & $<0.0001$ \\
\hline Hematoma/seroma & 1087 & 5262 & 0.52 & 251 & 1225 & 1.31 & $<0.0001$ \\
\hline Puncture vessel/nerve & 674 & 3274 & 0.32 & 155 & 759 & 0.81 & $<0.0001$ \\
\hline Wound dehiscence & 44 & 214 & 0.02 & 71 & 350 & 0.37 & $<0.0001$ \\
\hline Infection & 209 & 1026 & 0.1 & 166 & 814 & 0.87 & $<0.0001$ \\
\hline Other & 802 & 3913 & 0.39 & 295 & 1434 & 1.53 & $<0.0001$ \\
\hline Medical complication & 77 & 367 & 0.04 & 41 & 204 & 0.22 & $<0.0001$ \\
\hline
\end{tabular}

$\mathrm{F}=$ frequency; $\mathrm{WF}=$ weighted frequency. 